\title{
Anemia hemolítica autoinmune inducida por fármacos, como efecto adverso a vacuna frente a COVID-19, descripción de 1 caso y revisión de la bibliografía
}

\author{
Drug-induced autoimmune hemolytic anemia, as an adverse effect \\ to a vaccine against COVID-19, description of 1 case and review of the literature
}

\author{
Alejandra Canoa Rico ${ }^{1}$, Antonio García Ferreiro ${ }^{1}$, Samuel Roriguez Zorilla², Angel Martínez González \\ ${ }^{1}$ Hospital Ribera Povisa, Vigo. ${ }^{2}$ Universidad de Santiago de Compostela
}

\section{RESUMEN}

La anemia hemolítica autoinmune (AHAl) inducida por fármacos es un trastorno hematológico en cual se produce una destrucción inmnunitaria de los glóbulos rojos por anticuerpos. Los agentes más asociados son antibióticos y algunos quimioterápicos, pero también se han comunicado casos asociados con diferentes vacunas, incluido tras las vacunas frente a la COVID 19. Presentamos un caso de AHAl cuyo probable desencadenante fue la vacuna COVID 19 Vaxzevria.

Palabras clave: Anemia hemolitica autoinmune, Vacuna covid-19, Vaxzevria

La anemia hemolítica autoinmune (AHAl) inducida por fármacos es un trastorno hematológico en el cual se produce una destrucción inmunitaria de los glóbulos rojos generalmente mediada por anticuerpos. En la mayoría de los casos, los eritrocitos unidos a anticuerpos son fagocitados por macrófagos reticuloendoteliales en el bazo y/o hígado, lo que produce una hemólisis extravascular. Los fármacos más asociados a este proceso son algunos antibióticos (cefalosporinas o penicilinas), AINES y agentes quimioterápicos ${ }^{1,2}$. La presentación típica de AHAI inducida por medicamentos suele ser un proceso agudo producido en las siguientes horaso días tras la administración del fármaco. No existe evidencia de una asociación causal entre vacunas y la anemia hemolítica autoinmune, aunque sí se han reportado casos de AHAl tras la administración de ciertas vacunas $^{3,4}$.

Desde el inicio de la vacunación para SARS-CoV2 se han ido registrandocasos con diferentes efectos adversos. Entre los que han tenido mayor repercusión se encuentra la trombosis trombopénicainmune ${ }^{5,6}$. Hasta el momento, se han reportado varios casos de anemia hemolítica autoinmune relacionada con la vacunación ${ }^{7,8}$, que resumimos en la tabla 1.

Se han encontrado anticuerpos dirigidos contra la membrana eritrocitaria en la infección por SARS-CoV-2 y existen estudios que describen la asociación entre la infección por SARS-CoV-2 y la anemia hemolítica autoinmune ${ }^{9}$.

Presentamos el $1^{\circ}$ caso de anemia hemolítica autoinmune con antecedente de vacunación para COVID-19 con la vacuna vaxzevria como posible evento desencadenante.

\section{CASO 1}

Varón de 63 años como único antecedente de interés destaca ser fumador de 1 paquete/día desde los 44 años.

Acude a Urgencias por cuadro de 2 semanas de evolución de astenia progresiva, ictericia, orina anaranjada, pérdida de apetito y

\section{ABSTRACT}

Drug-induced autoimmune hemolytic anemia (AlHA) is a hematological disorder in which an immune destruction of red blood cells by antibodies occurs. Frequent agents that produce this disorder are antibiotics and chemotherapeutic agents; cases associated with different vaccines have been reported, including COVID-19 vaccines. We report a AHAI probably induced by the COVID-19 Vaxzevria vaccine.

Keywords: Autoimmune hemolytic anemia, Covid-19 vaccine, Vaxzevria

Tabla 1

\begin{tabular}{|c|c|c|c|}
\hline Variables & Caso 1 & Caso 2 & Caso 3 \\
\hline Sexo & Varón & Varón & Varón \\
\hline $\begin{array}{l}\text { Edad al } \\
\text { diagnostico (años) }\end{array}$ & 63 & 88 & 84 \\
\hline $\begin{array}{l}\text { Tipo de vacuna } \\
\text { Covid-19 }\end{array}$ & Vaxzevria & Cominarty & Cominarty \\
\hline $\begin{array}{l}1^{\mathrm{a}} 02^{\mathrm{a}} \text { dosis } \\
\text { vacuna Covid-19 }\end{array}$ & $1^{\circ}$ dosis & $2^{\circ}$ dosis & $1^{\circ}$ dosis \\
\hline $\begin{array}{l}\text { Relación temporal } \\
\text { con vacuna } \\
\text { Covid-19 }\end{array}$ & $\begin{array}{c}4 \text { días } \\
\text { después }\end{array}$ & 2 días después & $\begin{array}{l}19 \text { días } \\
\text { después }\end{array}$ \\
\hline $\begin{array}{l}\text { Signos/síntomas } \\
\text { clínicos }\end{array}$ & $\begin{array}{c}\text { Ictericia } \\
\text { Coluria } \\
\text { Astenia } \\
\text { Anorexia } \\
\text { Perdida } \\
\text { ponderal }\end{array}$ & $\begin{array}{c}\text { Ictericia } \\
\text { Coluria } \\
\text { Insomnio }\end{array}$ & $\begin{array}{c}\text { Ansiedad } \\
\text { Vértigo } \\
\text { Anorexia } \\
\text { Ictericia } \\
\text { Coluria } \\
\text { Dolor torácico. } \\
\text { Palpitaciones }\end{array}$ \\
\hline $\begin{array}{l}\text { Estudio } \\
\text { inmunologico }\end{array}$ & $\begin{array}{l}\text { IgG positivo } \\
\text { C3 d negativo }\end{array}$ & $\begin{array}{l}\text { IgG positivo } \\
\text { C3 d positivo }\end{array}$ & $\begin{array}{l}\text { IgG positivo } \\
\text { C3 d negativo }\end{array}$ \\
\hline
\end{tabular}

pérdida ponderal (5 kgs aproximadamente).Cuatro días antes del inicio de los síntomas recibió la primera dosis de Vaxzevria. En las pruebas complementarias realizadas al ingreso destacó una anemia hemolítica autoinmune $(\mathrm{Hb} 6.2 \mathrm{~g} / \mathrm{dL}, \mathrm{LDH} 485 \mathrm{U} / \mathrm{L}$, Bilirrubina total $4.56 \mathrm{mg} / \mathrm{dL}$ (Bilirrubina indirecta $3.27 \mathrm{mg} / \mathrm{dL}$ ), test de COOMBS +++ ) con reticulocitos disminuidos $(0.2 \%)$ y perfil férrico alterado 
(hierro y ferritina elevados, transferrina disminuida).Ingresó para estudio donde se completaron estudios analíticos presentando una proteína C reactiva elevada $(2.9 \mathrm{mg} / \mathrm{dL}$ ) y una $\lg \mathrm{G}$ e lgM disminuidas; el resto de los resultados fueron anodinos (leucocitos, creatinina, enzimas hepáticas, ácido fólico, vitamina B12, ANAs, complemento, IgG, proteinograma, Mantoux, serologías de Parvovirus, VHB, VHC y VIH negativos). Se realizó una TC toraco-abdomino-pélvica que descartó la presencia de un proceso tumoral asociado.

Se inició tratamiento con corticoterapia a dosis altas (1 mg/Kg/día), Rituximab semanal (4 dosis) e inmunoglobulinas IV con respuesta favorable. Actualmente tiene cifras de $\mathrm{Hb}$ en torno a $15 \mathrm{~g} / \mathrm{dL}$. El diagnóstico fue de anemia hemolítica autoinmune probablemente secundaria a vacuna Vaxzevria.

\section{CONCLUSIONES}

Las vacunas frente a la COVID 19 han demostrado su eficacia y seguridad en la prevención de la enfermedad, así como en la reducción de la morbimortalidad ${ }^{10-12}$. En comparación con los beneficios, son mínimos los efectos adversos descritos, pero deben ser tenidos en cuenta de cara a un diagnóstico acertado que disminuya la morbimortalidad de estos.

Aunque la mayoría de las anemias hemolíticas autoinmunes acaban siendo catalogadas como idiopáticas, el hecho de que el virus SARS-CoV-2 se haya asociado con AHAI hacen pensar que en este caso el proceso podría tener relación con la vacunación recibida 4 días antes, por lo que consideramos que debería de ser incluida en ficha técnica.

\section{FINANCIACIÓN}

Este trabajo no ha recibido ningún tipo de financiación.

\section{CONFLICTO DE INTERESES}

Los autores declaran no tener ningún conflicto de intereses.

\section{BIBLIOGRAFIA}

1. Garbe E, Andersohn F, Bronder E, Klimpel A, Thomae M, Schrezenmeier H, et al. Drug induced immune haemolyticanaemia in the Berlin Case-Control Surveillance Study. Br J Haematol. 2011;154(5):644-653.

2. Johnson ST, Fueger JT, Gottschall JL. One center's experience: the serology and drugs associated with drug-induced immune hemolytic anemia - a new paradigm. Transfusion. 2007;47(4):697-702.

3. Shizuma T. Autoimmune hemolytic anemia following influenza virus infection or administration of influenza vaccine. J Blood Disorders Transf. 2014;5(3):1000200.

4. Montagnani S, Tuccori M, Lombardo G, Testi A, Mantarro S, Ruggiero E, et al. Autoimmune hemolytic anemia following MF59-adjuvanted influenza vaccine administration: a report of two cases. Ann Pharmacother. 2011;45(1):e8.

5. Marcucci R, Marietta M. Vaccine-induced thrombotic thrombocytopenia: the elusive link between thrombosis and adenovirus-based SARS-CoV-2 vaccines. Internal and Emergency Medicine. 2021:1-7.

6. Tiede A, Sachs UJ, Czwalinna A, Werwitzke S, Bikker R, Krauss JK, et al. Prothrombotic immune thrombocytopenia after COVID-19 vaccination. Blood, The Journal of the American Society of Hematology. 2021;138(4):350-353.

7. Brito S, Ferreira N, Mateus S, Bernardo M, Pinto B, Lourenço A, et al. A Case of Autoimmune Hemolytic Anemia Following COVID-19 Messenger Ribonucleic Acid Vaccination. Cureus. 2021;13(5).

8. Murdych TM. A case of severe autoimmune hemolytic anemia after a receipt of a first dose of SARS-CoV-2 vaccine. International Journal of Laboratory Hematology. 2021;10.1111/ijlh.13653.

9. Algassim AA, Elghazaly AA, Alnahdi AS, Mohammed-Rahim OM, Alanazi AG, Aldhuwayhi NA, et al. Prognostic significance of hemoglobin level and autoimmune hemolytic anemia in SARS-CoV-2 infection. Ann Hematol. 2021;100(1):37-43.

10. Polack FP, Thomas SJ, Kitchin N, Absalon J, Gurtman A, Lockhart S, et al. Safety and efficacy of the BNT162b2 mRNA Covid-19 vaccine. N Engl J Med. 2020;383(27):2603-2615

11. Baden LR, El Sahly HM, Essink B, Kotloff K, Frey S, Novak R, et al. Efficacy and safety of the mRNA-1273 SARS-CoV-2 vaccine. N Engl J Med. 2021;384(5):403416

12. Voysey M, Clemens SAC, Madhi SA, Weckx LY, Folegatti PM, Aley PK, et al. Safety and efficacy of the ChAdOx1 nCoV-19 vaccine (AZD1222) against SARS-CoV-2: an interim analysis of four randomised controlled trials in Brazil, South Africa, and the UK. TheLancet. 2021;397(10269):99-111. 